\title{
Fragmentation spectroscopy of heterogeneous clusters
}

\author{
E. Holub-Krappe, G. Ganteför *, G. Bröker, and A. Ding \\ Bereich Strahlenchemie, Hahn-Meitner-Institut, D-1000 Berlin 39, Germany
}

Photoionisation experiments were performed with heterogeneous Ar-Xe-clusters produced by supersonic expansion of argon gas with small quantities of xenon added to it. A threshold-electron photoionisation (TEPICO) technique was used to obtain time of flight cluster mass spectra. These mass spectra show particularly strong intensities for $\mathrm{Ar}_{12} \mathrm{Xe}^{+}$and $\mathrm{Ar}_{18} \mathrm{Xe}^{+}$which are attributed to the extraordinary stabilities of these cluster ions. Maxima in the ionic size distribution around $\mathrm{Ar}_{7} \mathrm{Xe}^{+}$are related to a particular abundance of neutral $\mathrm{Ar}_{12} \mathrm{Xe}$ which is fragmented after ionization. These stabilities are explained in terms of geometries consisting of a central Xe atom or ion surrounded by shells of Ar atoms. Filled shells exhibit particular strong bonding because these exhibit the largest number of atom-atom bonds. This conclusion is supported by simple theoretical calculations. The ionization process is discussed in terms of two direct and one indirect ionization channels the latter one proceeding via an intermediate electronic excitation of the Ar component in the neutral cluster.

PACS: $33.65 ; 33.80 . \mathrm{E} ; 36.40$

\section{Introduction}

Otto Stern's pioneering experiments laid the ground work for generations of atomic and molecular beam experiments, which by now have become a standard tool for the experimentalist [1]. The invention of the supersonic beam technique has made it possible to produce high density atomic and molecular beams [2]. Condensation processes in the expansion which lead to clusters in the beam were at first regarded as a disturbing and unwanted side effect to be avoided at all costs [3]. In the long run, they gave rise to the development of a complete new field of cluster experiments [4-9], which are able to probe the static and dynamic properties of the clusters in the beam. Even industrial applications have appeared in the last years $[10]$.

As early as 1956 experiments had been performed on rare gas clusters [4], but only through the introduction of more sophisticated experimental techniques has it been possible to perform quantitative

* Present address: Fakultät für Physik, Universität Bielefeld, D-4800 Bielefeld, Federal Republic of Germany experiments. One of the great obstacles to the interpretation of cluster experiments is the understanding of fragmentation, as this plays a major role in most processes investigated so far. In particular, there is an urgent need for experiments which investigate such processes under well defined initial conditions.

There have been many investigations in the field of laser induced fragmentation where clusters dissociate after absorbing photons of known energy [11]. Such techniques provide information on how the energy levels of clusters depend on their size. A drawback to these methods is, however, that they are not size selective for neutral clusters. The ultimate goal is to perform experiments with size selected neutral cluster beams, which would give insight into the dependence of energy states as a function of cluster size. At present such experiments are not technically feasible.

Fragmentation is also of great importance in all types of experiments involving ionization of cluster beams. Usually the ionization of clusters is followed by changes in the geometrical configuration, with a concomitant release of internal energy. This leads to fragmentation of the weakly bound cluster with the 
consequence that the measured ionic size distribution does not resemble the neutral one [12]. Fragmentation is therefore a great obstacle to the understanding of cluster properties.

The effect of ionization-induced fragmentation is particularly pronounced in weakly bound van-derWaals molecules and has given rise to many controversial debates $[12,13]$. It is now generally believed that ionization accompanied by fragmentation is the rule rather than the exception. In fact, it is agreed that the resulting mass spectrum is a convolution of the neutral distribution with the fragmentation probability of the single clusters. It is, however, still a matter of speculation as to which of these contributes more, i.e. if the measured mass spectrum is dominated by the fragmentation process or if it resembles the neutral distribution. In order to shed light on these important processes, it is necessary to perform very detailed experiments under known initial conditions.

One important prerequisite for the experiments is a knowledge of the amount of energy dissipated during the ionization process. In most of the experimental techniques, the available energy is divided amongst the different particles, the exact distribution being unknown. The system most desirable would be one, where the kinetic energy of all particles involved could be determined with sufficient accuracy. Only then the exact amount of energy dissipated in the cluster ion would be known and could be used to elucidate the fragmentation dynamics of the ion in a quantitative way. Because of the well defined energy of the ionizing photon it is sufficient to measure the kinetic energy of the electron. Unfortunately, usual kinetic energy analyzers for electrons are not well suited to this type of experiments: time-of-flight techniques require a pulsed photon source; electrostatic analyzers have insufficient transmission to be used in experiments with free cluster beams.

Therefore we have applied the TEPICO (Threshold Electron Photo Ion COincidence) method [14, $15]$ to this type of cluster experiment: The mass spectra of the cluster ions are measured using a time of flight technique in coincidence with the electrons which have kinetic energies of approximately zero. By being limited to zero energy electrons not all processes will be detected and so only a selected number of systems can be analyzed: this can be done, however, in much greater detail compared with other conventional methods. Furthermore, the experiment has been devised in such a way which allows the determination of the fragmentation induced kinetic energy release of the cluster ion.

In the case of van-der-Waals clusters, the ionization process results in a large change in cluster geometry. In heterogeneous systems these changes are much

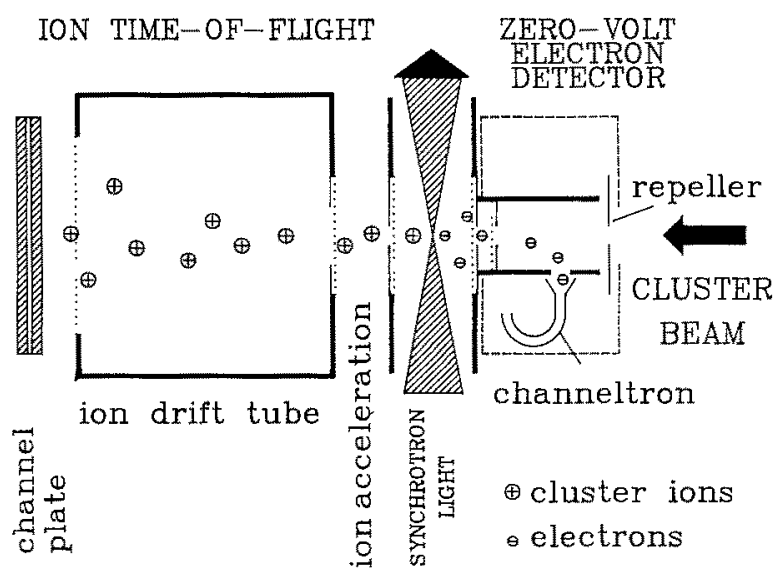

Fig. 1. Schematic experimental set-up

smaller than in homogeneous systems (a similar effect is obtained for homonuclear and heteronuclear rare gas molecules [16].

To facilitate interpretation of the experimental data, we have centred our attention on clusters of the type $X_{n} Y$ where $X$ and $Y$ are rare gas atoms, $Y$ having the lower ionization potential. This choice will ensure that the charge will finally reside on atom $Y$. Dimer or trimer formation will not affect the results in such cases [17-19].

\section{Experimental}

The experiments were performed in a two chamber molecular beam apparatus which has been described in principle in an earlier publication [15]. It is shown schematically in Fig. 1. The mixed clusters were generated by expanding a mixture of $0.4 \% \mathrm{Xe}$ in $\mathrm{Ar}$ through a $30 \mu \mathrm{m}$ diameter nozzle. After passing a skimmer, the cluster beam was then crossed with the monochromatized light from the Berlin Electron Synchrotron facility BESSY. Ions and electrons formed in the ionization process were extracted in opposite directions collinear to the neutral beam. A zero-volt electron analyser was used to detect only those electrons below a threshold energy set by the magnitude of the electrostatic extraction field. The time-of-flight between the arrival of the zero volt electron and the cluster ion was recorded in a multichannel analyser.

The method functions properly if only one ionelectron pair is in the drift tube at a time, which limits the maximum count rate. By rule of thumb, it was found that ion count rates of $0.05 \mathrm{f}$ can be used without significant deterioration of the signal, $f$ being the inverse of the flight time of the heaviest cluster ion. If ion intensities are too high, random coincidences 

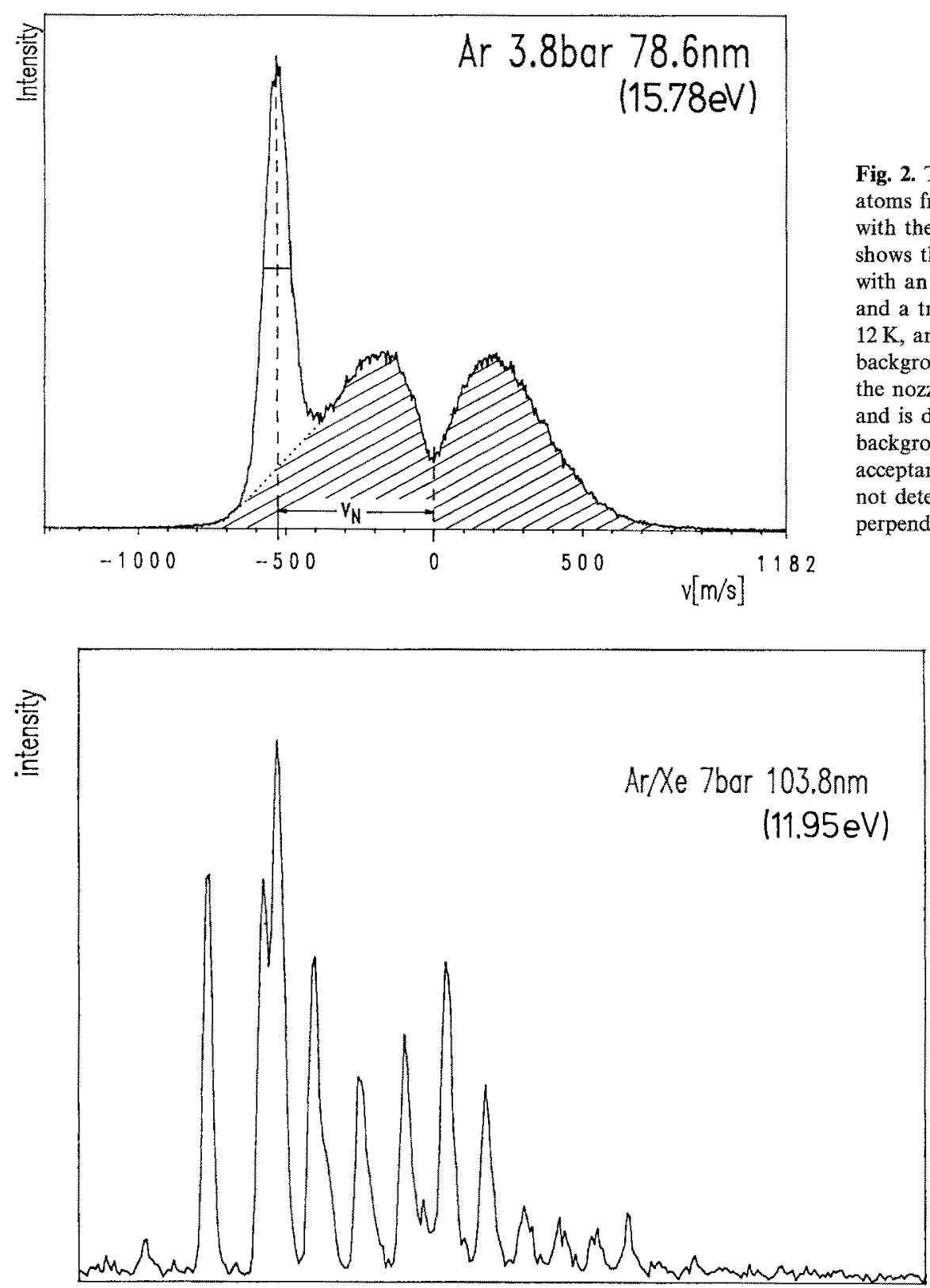

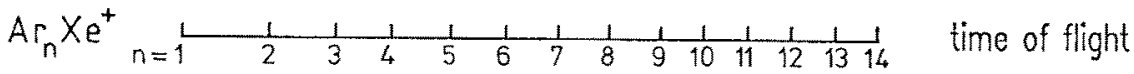

$A r_{n} X e_{2}^{+} n=0 \quad \frac{1}{1}-\frac{1}{2} \quad \frac{1}{3}$

of ions and electrons arising from different clusters produce an undesirable statistical background.

Two different forms of the extraction field were used for the ions: One (I) consists of a constant extraction field (length: $b$, field strength: $E$ ) followed by a zero drift-field (length: $d$ ); the other (II) set-up consists of two electrostatic fields (length: $b_{1}, b_{2}$ ) with different field strengths $\left(E_{1}, E_{2}\right)$ followed by a field free region (length: $d$ ). The latter set-up uses very short acceleration distances and therefore short accelera-
Fig. 2. Typical time-of-flight spectrum of Ar atoms from a supersonic nozzle source taken with the TEPICO-method. The distribution shows the supersonic Ar beam (narrow peak) with an average translational energy of $58 \mathrm{meV}$ and a translational temperature smaller than $12 \mathrm{~K}$, and thermal Ar-atoms from the background gas (shaded area). The velocity of the nozzle beam was determined to $530 \mathrm{~m} / \mathrm{s}$ and is denoted by $v_{n}$. The dip in the background distribution is caused by the finite acceptance angle of the detector, which does not detect particles having too large velocities perpendicular to the beam axis
Fig. 3. Typical time-of-flight spectra of $\mathrm{Ar}_{n} \mathrm{Xe}^{+}$cluster ions generated by an expansion of a gas mixture of $0.4 \%$ Xe in Ar through a $30 \mu \mathrm{m}$ nozzle. Small contributions from the $\mathrm{Ar}_{n} \mathrm{Xe}_{2}$ series are also visible. The shape of the $\mathrm{Ar}_{12} \mathrm{Xe}^{+}$peak consisting of a narrow and a broad distribution should be noted tion times. In both cases the ratios of the field strengths and lengths were optimised to achieve time focussing for ions formed in different parts of the ionization region. The best focussing conditions require rather large extraction fields (a few hundred $\mathrm{V} / \mathrm{cm}$ ) which are quite different from the low fields used for electron extraction. Therefore a pulse technique was applied where the appropriate extraction field changes from low to high, after the electron has been detected. The best resolution obtained in this type 


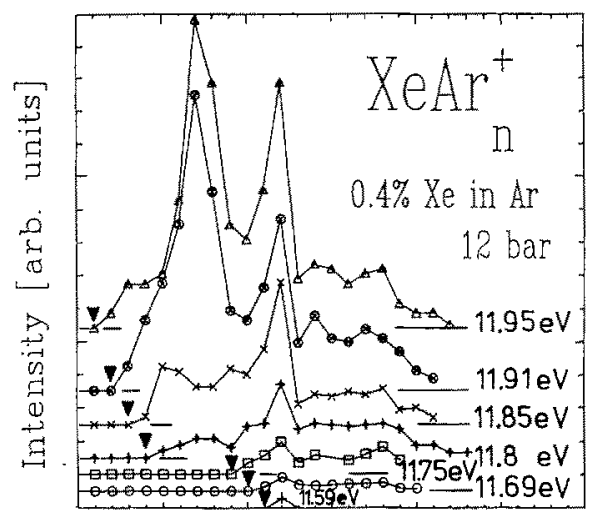

$\begin{array}{lllllll}0 & 5 & 10 & 15 & 20 & 25 & 30\end{array}$

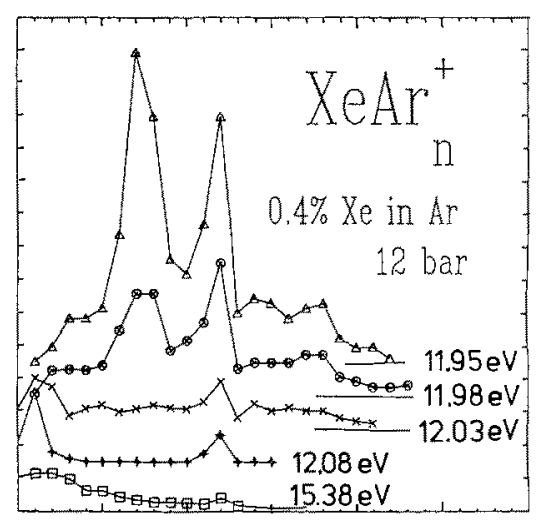

$5101520 \quad 25 \quad 30$
Fig. 4. Size distribution of the $\mathrm{Ar}_{n} \mathrm{Xe}^{+}$-cluster ions as a function of photon energy. The arrows denote the appearance of the smallest cluster for a given energy, the horizontal lines indicate the zero intensity for a particular cluster ion distribution

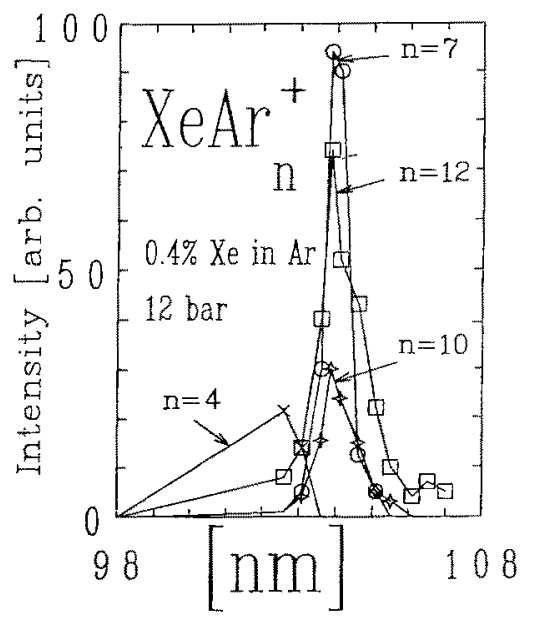

Fig. 5. Relative intensities for some selected $\mathrm{Ar}_{n} \mathrm{Xe}^{+}$-cluster ions as a function of photon energy

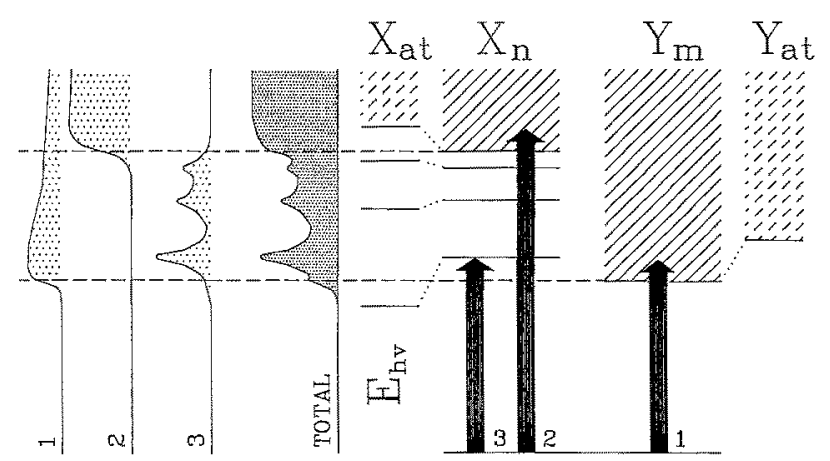

Fig. 6. Schematic diagram of the energy levels of a mixed cluster $X_{n} Y_{m}$ (right) and its ionisation efficiency curve (left). The energy levels (the ionization continuum is hatched) of the cluster components $\left(X_{n}, Y_{m}\right)$ are shifted in respect to the atomic ones $\left(X_{\mathrm{at}}, Y_{\mathrm{at}}\right)$. Only such excitations which lie above the lowest ionic level $(--)$ will contribute to ionization. The hole will be finally trapped in the lowest energy level possible, i.e. at the ionization threshold of $Y_{m}$ of experiment is $\Delta E=25 \mathrm{meV}$ for the electrons and $m / \Delta m=500$ for the mass resolution. In exceptional cases (ionization of the Ar monomer), coincidence rates of up to $25 \%$ were obtained corresponding to a detection efficiency of at least $50 \%$ for both electrons and ions.

If ions fragment very shortly after ionization, the kinetic energy released gives rise to an initial velocity $v_{0}$ which changes the flight time in the following manner

$t_{I}=\frac{m}{e E}\left(v_{t}-v_{0}\right)+\frac{d}{v_{t}}$

$v_{t}=\left(\frac{2 b}{m}+v_{0}^{2}\right)^{\frac{1}{2}}$

$t_{I I}=\frac{m}{e E_{1}}\left(v_{t t}-v_{t}\right)+\frac{m}{e E_{2}}\left(v_{t t}-v_{t}\right)+\frac{d}{v_{t t}}$

$v_{t}=\left(\frac{2 b_{1}}{m}+v_{0}^{2}\right)^{\frac{1}{2}}$

$v_{t t}=\left(\frac{2 b_{2}}{m}+v_{t}^{2}\right)^{\frac{1}{2}}$.

As the direction of the fragmentation products is randomly distributed in space, this leads to a broadening of the cluster ion peak, which is used for the quantitative determination of the kinetic energy release from fragmentation. Sometimes, the cluster-ion peak revealed a dip in the centre, if the energy released was too large (see Fig. 2). This removed the sideways scattered fragment ions out of the acceptance angle of the detector.

If cluster ions fragment in the acceleration region, they have flight times lying between those of the unfragmented and fragmented cluster ion [20]. It is therefore possible to analyse the fragmentation of the cluster ions in terms of energy release and lifetime of the metastable parent ions. 


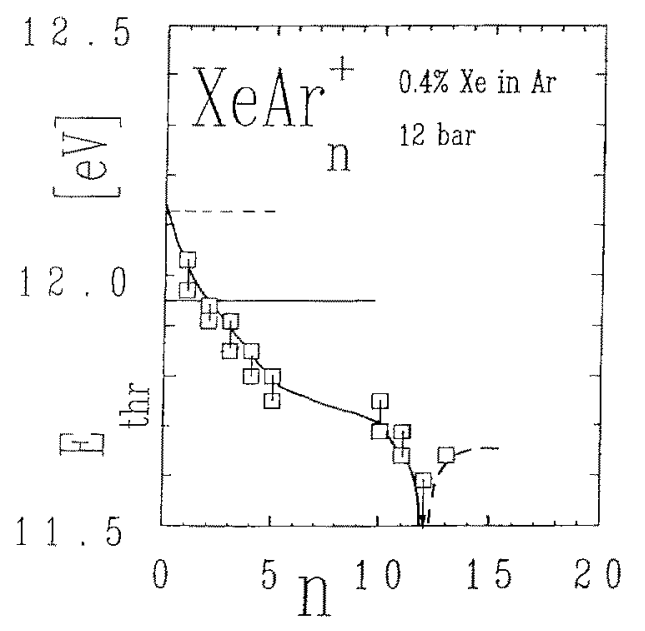

Fig. 7. Threshold photon energies $\left(E_{\mathrm{thr}}\right)$ for the appearance of clusters $\mathrm{Ar}_{n} \mathrm{Xe}^{+}$as a function of the number of $\mathrm{Ar}$ atoms $n$ in the cluster. The dashed horizontal line denotes the ionization potential of $\mathrm{Xe}$, the solid line the energy at which the resonance appears

\section{Results and discussion}

Time-of-flight spectra of photoionized cluster ions were measured at different photon energies and under various expansion conditions. Typical TEPICO spectra are shown in Fig. 3. The size dependent structure of the cluster ions is clearly visible, particularly the intensity maxima for $\mathrm{Ar}_{12} \mathrm{Xe}^{+}$. Besides the sequences containing a single $\mathrm{Xe}$ atom (or ion) a second series of the composition $\mathrm{Ar}_{n} \mathrm{Xe}_{2}^{+}$is also detectable. The gas mixing ratio had been adjusted such as to maximize the formation of $\mathrm{Ar}_{n} \mathrm{Xe}^{+}$while keeping the $\mathrm{Ar}_{n} \mathrm{Xe}_{m}^{+}(m \geqq 2)$ component small. By increasing the fraction of $\mathrm{Xe}$ in the expansion mixture a large range of $\mathrm{Ar}_{n} \mathrm{Xe}_{m}^{+}$cluster ions could be generated.

The mass spectra show a strong wavelength dependence (see Fig. 4). Particularly noticeable is the appearance of a third maximum in the size distribution for photon energies between $11.80 \mathrm{eV}(105.1 \mathrm{~nm})$ and $11.98 \mathrm{eV}(103.6 \mathrm{~nm})$. Figure 5 displays the intensity of a particular cluster ion peak as a function of photon energy. It clearly shows a single narrow resonance-like structure around $11.95 \mathrm{eV}(103.85 \mathrm{~nm})$. Not displayed in this figure is the increase in intensity around the direct ionization threshold of the Ar compounds (appr. $15 \mathrm{eV}$ ). In spite of the very narrow structure, different peak shapes appear for $\mathrm{Ar}_{n} \mathrm{Xe}^{+}$ when $n<10$ and $n \geqq 10$, the latter ones displaying a slight shoulder towards smaller energies (see Fig. 5).

Similar behaviour was found for a series of mixed cluster systems $[21,22]$. It is widely believed that ionization in a mixed system of composition $X_{n} Y_{m}$ ( $X, Y$ atoms or molecules, $Y$ having the lower ionization potential) proceeds via three pathways (Fig. 6)
1) direct ionization of the $Y$-component;

2) direct ionization of the $X$-component and subsequent charge transfer to the $Y$-component accompanied by the release of the surplus energy into internal degrees of freedom;

3 ) excitation of the $X$-component into a Rydberglike state and subsequent energy transfer to the $Y$ component followed by removal of the electron from the cluster system.

While ions are always formed in the cases 1) and 2) (the electron has already been removed in the first step of the process), ion formation in case 3 ) is only one of many channels. It is also possible to produce a non-ionized cluster compound from the excited intermediate state by energy transfer, the surplus energy being used to fragment the neutral cluster. It is not possible to estimate these contributions, as our experiment is not sensitive to neutral clusters. Photoionization efficiency curves almost always show the direct ionization channels, each of which appears as a step function in the photoionization spectrum. Both can be observed even if one component is lost through subsequent fragmentation $[21,23]$.

While processes 1) and 2) are always found in mixed cluster systems, process 3 ) is not always detected. Many systems [24] do not exhibit any resonance behaviour at all, while some show one line [25] and others several resonance lines [26]. According to the foregoing discussion, one would attribute this to energy paths which are different for each system depending on the structure of the molecular orbitals. This is in some respects similar to curve crossing processes in diatomic and polyatomic systems, where the transition probability depends very much on the local structure of the potential curves involved. In cases where $X$ is a rare gas, the resonance structure can be attributed to a Rydberg state which is shifted from its atomic position by interaction with neighbouring cluster particles.

Similar effects have been observed in the solid state and in matrix isolation studies [27], where the shift of the particular state is attributed to the repulsion of the surrounding atoms. In the case of an excited cluster however, effects like polarization forces have to be taken into account, which would cause a similar shift in energy levels. Until now, only shifts towards shorter wavelength have been observed in matrix isolation experiments. The cage effect, as observed in matrix isolation experiments [28] predicts a larger shift for the excited state thus causing a shift towards shorter wavelengths (larger energies). The effect of polarisation forces would produce a slightly smaller equilibrium geometry of the excited state and therefore shift the levels towards larger energies. Both effects depend on the position of the excited particle 


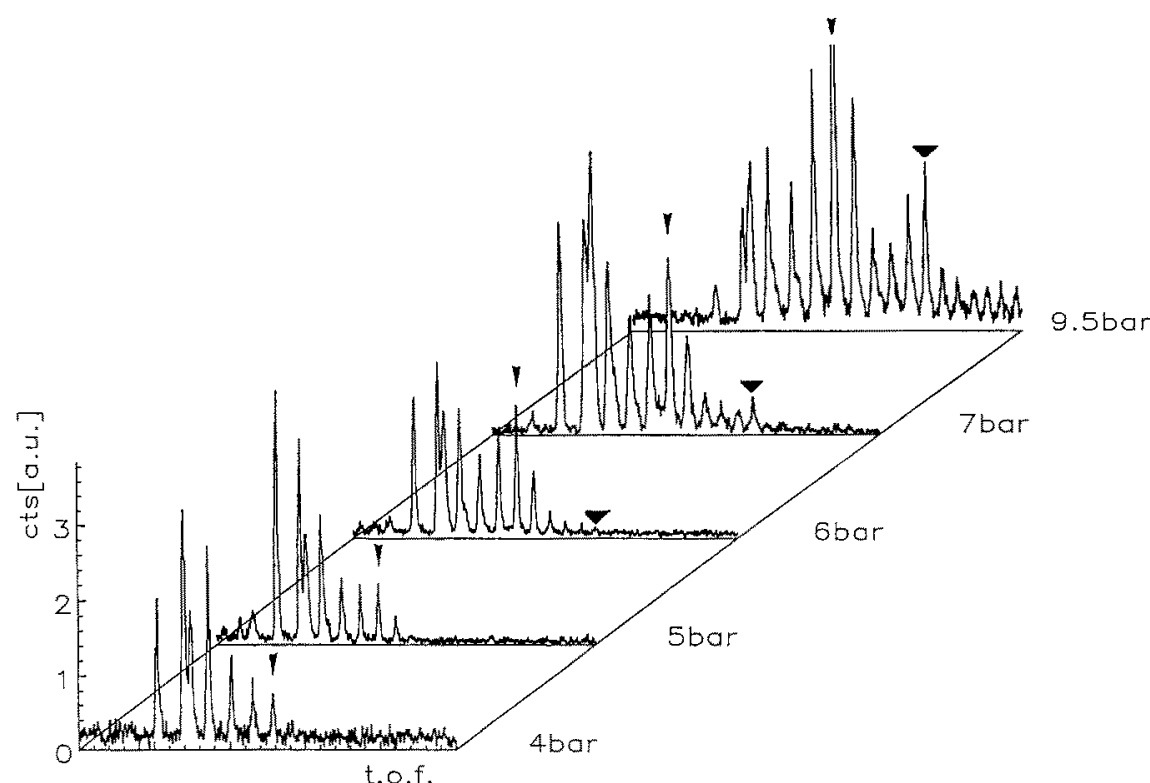

Fig. 8. $\mathrm{Ar}_{n} \mathrm{Xe}^{+}$distribution as a function of stagnation pressure. $\mathrm{Ar}_{7} \mathrm{Xe}^{+}$ is marked by a small, $\mathrm{Ar}_{12} \mathrm{Xe}^{+}$by a broad arrow. The photon energy is $11.95 \mathrm{eV}(\lambda=103.8 \mathrm{~nm})$ within the cluster and will appear strongest when it is in the centre and weakest for locations near the surface of the cluster. This could provide a tool for evaluating the position of the excited particle in the cluster. In the present case the component $X$ is the Ar atom, and $Y$ is Xe.

The different shapes of the resonance line which are observed for $\mathrm{Ar}_{7} \mathrm{Xe}^{+}$and $\mathrm{Ar}_{12} \mathrm{Xe}^{+}$are attributed to the difference in the number of shells of the neutral excited precursor and will be discussed later.

The appearance energy, i.e. the lowest energy at which a particular cluster ion is observed, is marked by black arrows in Fig. 4 and is displayed as a function of the cluster ion size in Fig. 7. Surprisingly, this function is non-linear and non-monotonic. In particular, $\mathrm{Ar}_{12} \mathrm{Xe}^{+}$has the lowest appearance energy, which is even lower than that of larger clusters. This is unexpected as the total binding energy is usually assumed to increase with cluster size [19]. The measured thresholds, however, do not necessarily have to agree with the adiabatic ionization potential. Because of the change in geometry, the Franck-Condon factors for adiabatic ionization are rather small, which makes a direct transition into the ground state of the cluster ion rather improbable. A lower ionization threshold, as compared to larger and smaller clusters, could therefore be interpreted in terms of more favourable Franck-Condon factors, implying only a small change between the neutral and ionic equilibrium geometry for this particular cluster. This is also in agreement with the appearence of long lived metastable $\mathrm{Ar}_{12} \mathrm{Xe}^{+}$cluster ions (to be discussed later).

In order to clarify this matter, a series of experiments with varying expansion conditions have been performed (see Fig. 8). On lowering the expansion pressure the spectrum changes significantly: the maximum around $\mathrm{Ar}_{7} \mathrm{Xe}^{+}$becomes weaker and finally disappears altogether. At the same time, the peak shapes of several larger cluster-ions become narrower and display a structure which consists of a narrow and a broad distribution. The question arises whether experiments like the ones described above can elucidate the cluster geometry or other cluster properties. Electron diffraction [8] and mass spectrometric experiments $[29,30]$ suggest - sometimes wrongly - that particular cluster species exhibit geometries which are highly symmetric and feature a central atom (or ion) surrounded by shells of atoms. Clusters with completely filled shells are particularly stable; they are described in terms of "magic" numbers [29], an expression borrowed from theory of nuclear structure [31]. Simple models which count merely the number of bonds in a cluster will justify these assumptions. Mixed systems of the type $X_{n} Y$ show an even more pronounced effect as one has to take into account the different bond strength between equal atoms $(X-X)$ and non equal atoms $(X-Y)$. The latter ones have usually a larger binding energy in neutral vander-Waals systems.

The most stable geometry for both neutral and ionic systems is the one where $Y$ is the central atom surrounded by shells of atoms $X$. Atoms in the first shell are more strongly bound than those in the following shells, as they are in direct contact with the central hetero-atom (see Fig. 9). These qualitative findings are justified by detailed calculations which have been performed by different groups using DIM $[17]$ and $a b$ initio methods to determine the bond energy and the cluster geometry [18]. 


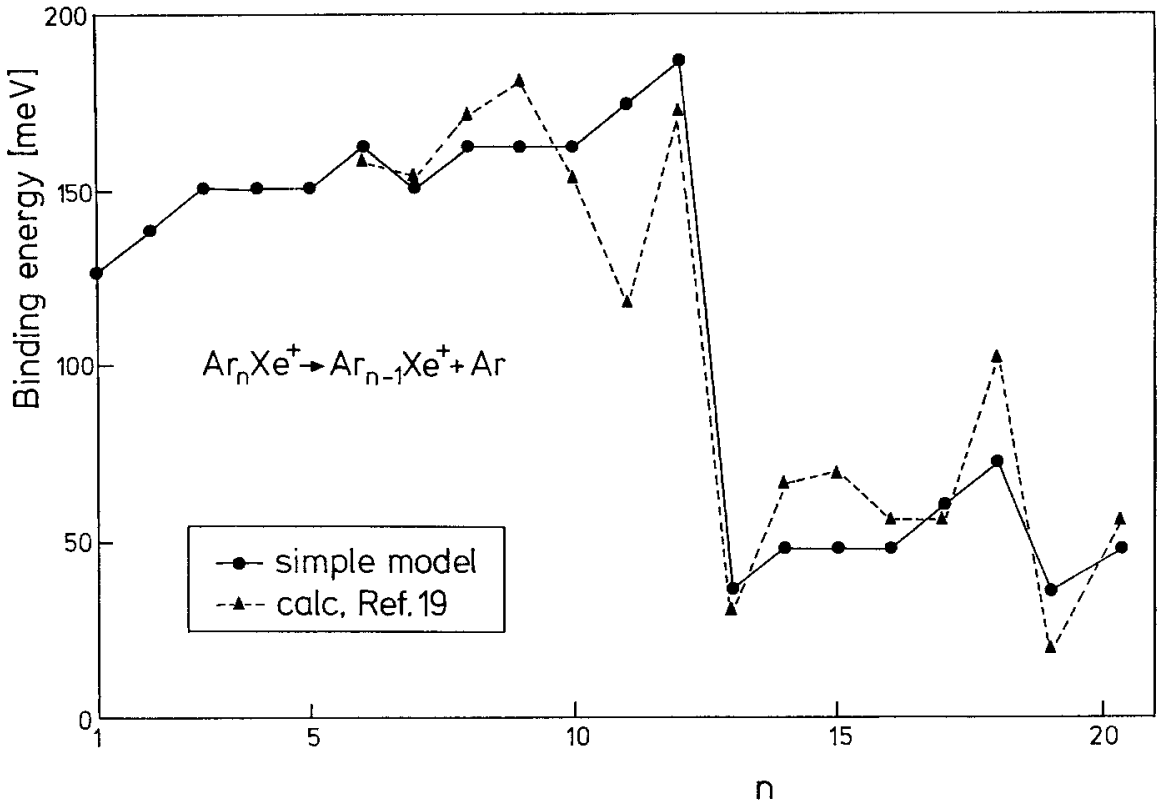

Fig. 9. The binding energy of Ar atoms in a $\mathrm{Ar}_{n} \mathrm{Xe}^{+}$cluster-ion according to a simple model and to calculations of Böhmer [19]. The simple model assumes pairwise additive potential functions where only such bonds between adjacent particles are taken into account. Bond energies of $127 \mathrm{meV}$ for the $\mathrm{Xe}^{+}$-Ar bond and $12 \mathrm{meV}$ for the $\mathrm{Ar}-\mathrm{Ar}$ bond have been used
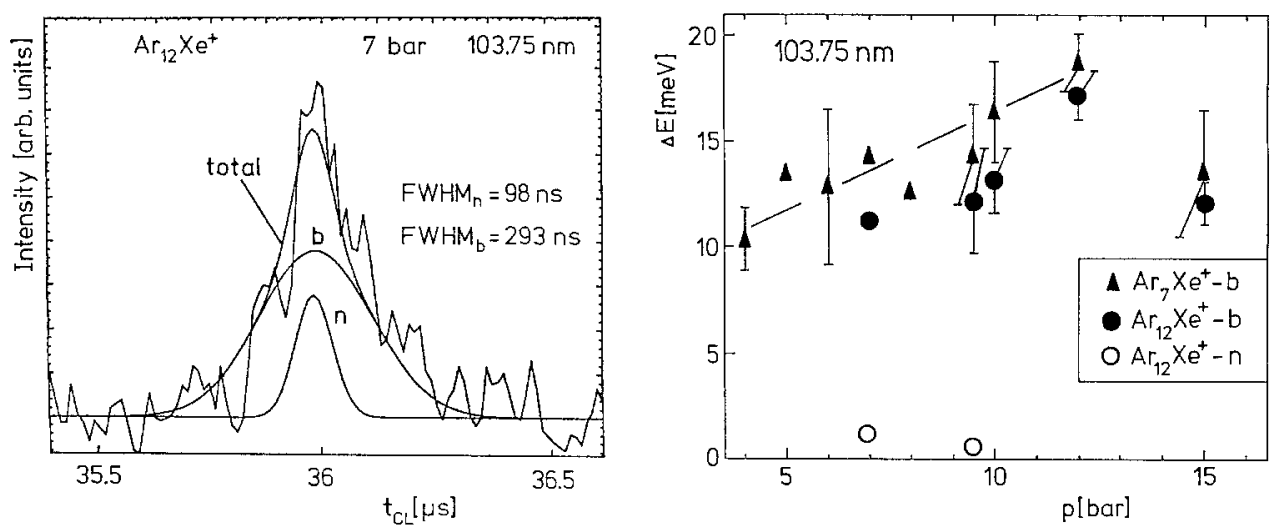

Fig. 10. Left: Line analysis of the $A r_{12} \mathrm{Xe}^{+}$cluster right: Fragmentation energy release $\Delta E$ as a function of pressure for $A r_{7} \mathrm{Xe}^{+}$and $\mathrm{AR}_{12} \mathrm{Xe}^{+}$. Symbols $n$ and $b$ denote the narrow and the broad components of the cluster peaks, respectively. The broken lines indicate the average release energy for the $\mathrm{Ar}_{7} \mathrm{Xe}^{+}$clusters. At low pressures ( $\left.p=6 \mathrm{bar}\right) \mathrm{Ar}_{12} \mathrm{Xe}$ is no longer produced. At high pressure ( $p=10 \mathrm{bar}$ ) the narrow component cannot be seen because of too large fragment ion intensity. The points at 15 bar have to be interpreted with caution as the molecular beam is at the verge of breakdown because of limited pumping speed

A particularly detailed theoretical study for the $\mathrm{Ar}-\mathrm{Xe}$ system has been performed by Böhmer and Peyerimhoff $[18,19]$, who used statistical methods to evaluate the bond strength in an $\mathrm{Ar}_{n} \mathrm{Xe}^{+}$ion. They find large binding energies for $\mathrm{Ar}_{12} \mathrm{Xe}^{+}, \mathrm{Ar}_{18} \mathrm{Xe}^{+}$ and $\mathrm{Ar}_{22} \mathrm{Xe}^{+}$as well as for $\mathrm{Ar}_{9} \mathrm{Xe}^{+}$. The stability of the former three is explained by closed shell geometries, that of the latter by the fact that in order to obtain the maximum coordination number of 12 , all $\mathrm{Ar}-\mathrm{Xe}$ bonds have to be slightly stretched; $\mathrm{Ar}_{9} \mathrm{Xe}^{+}$ is the largest cluster ion where all Ar atoms can be accomodated without stretching the $\mathrm{Ar}-\mathrm{Xe}$ bond [32].

The analysis of the line-shape of the ion time-offlight peaks reveals a broadening larger than that ex- pected from the intrinsic resolution of the apparatus (as checked with the monomer ion). This is evidence for kinetic energy release caused by fragmentation of larger species and has been observed in other cluster systems too [20]. The observed broadening appears to depend on the cluster ion size.

The energy available for fragmentation is the difference between the energy of the absorbed photon and the lowest ionization energy of the cluster, which is dissipated as internal energy (vibration, phonons). This gives rise to a kinetic energy release and therefore to a broadening of the time-of-flight peaks. Several cluster ion peaks, particularly $\mathrm{Ar}_{12} \mathrm{Xe}^{+}$, exhibit contributions from at least two processes causing different line widths (see Fig. 10). There is evidence that 
the broad base of the peak is caused by fragmentation from larger clusters; the narrow part being due to metastable ions formed without fragmentation from $\mathrm{Ar}_{12} \mathrm{Xe}$. Experiments performed under various expansion conditions (see Fig. 8) reveal that both features in the $\mathrm{Ar}_{12} \mathrm{Xe}^{+}$-peak can be seen using rather large stagnation pressures. Lowering the pressure, i.e. narrowing the neutral distribution, the broad shoulder weakens in favour of the narrow one until finally the narrow one is the only contribution to be seen. Lowering the pressure further, the narrow $\mathrm{Ar}_{12} \mathrm{Xe}^{+}$peak dissapears altogether; at the same time the maximum around $\mathrm{Ar}_{7} \mathrm{Xe}^{+}$in the ionic size distribution disappears.

This leads us to the following conclusions:

1. The maxima around $\mathrm{Ar}_{12} \mathrm{Xe}^{+}$and $\mathrm{Ar}_{18} \mathrm{Xe}^{+}$ are caused by particularly stable ionic configurations where the $\mathrm{Xe}^{+}$is surrounded by complete shells of Ar atoms. They are predominantly formed by ionization and subsequent fragmentation of larger clusters:

$$
\begin{aligned}
\mathrm{Ar}_{n} \mathrm{Xe} & +h v \rightarrow\left(\mathrm{Ar}_{n} \mathrm{Xe}\right)^{+*} \rightarrow \mathrm{Ar}_{12} \mathrm{Xe}^{+}+(n-12) \cdot \mathrm{Ar} \\
& +E_{\mathrm{kin}}, \\
\mathrm{Ar}_{n} \mathrm{Xe} & +h v \rightarrow\left(\mathrm{Ar}_{n} \mathrm{Xe}\right)^{+*} \rightarrow \mathrm{Ar}_{18} \mathrm{Xe}^{+}+(n-18) \cdot \mathrm{Ar} \\
& +E_{\mathrm{kin}} .
\end{aligned}
$$

2. The maxima around $\mathrm{Ar}_{7} \mathrm{Xe}^{+}$and the metastable part (narrow portion) of the $\mathrm{Ar}_{12} \mathrm{Xe}^{+}$have the neutral $\mathrm{Ar}_{12} \mathrm{Xe}$ as a common precursor:

$$
\begin{aligned}
& \nearrow \mathrm{Ar}_{6} \mathrm{Xe}^{+}+6 \mathrm{Ar}+E_{\mathrm{kin}} \mathrm{Xe}+h v \rightarrow\left(\mathrm{Ar}_{12} \mathrm{Xe}\right)^{+*} \\
& \rightarrow \mathrm{Ar}_{7} \mathrm{Xe}^{+}+5 \mathrm{Ar}+E_{\mathrm{kin}} \\
& \searrow \mathrm{Ar}_{8} \mathrm{Xe}^{+}+4 \mathrm{Ar}+E_{\mathrm{kin}} \\
& \rightarrow\left(\mathrm{Ar}_{12} \mathrm{Xe}\right)^{+(*)} \text { metastable. }
\end{aligned}
$$

The excess energy in process $(5 a)$ is dissipated by releasing approximately $5 \mathrm{Ar}$ atoms. This is also an explanation for the finding that the threshold energy of $\mathrm{Ar}_{n} \mathrm{Xe}^{+}$(c.f. Fig. 7) shows a non monotonic behaviour for $6 \leqq n \leqq 9$.

The result is not surprising as simple model calculations show that clusters of the type $X_{12} Y\left(\operatorname{Ar}_{12} \mathrm{Xe}\right)$ are also the most stable ones in the neutral system and are therefore expected to be the most abundant ones in the neutral beam.

The form of the observed resonance line shape (Fig. 5) can also be described by the same mechanism:

The resonance level correlates with the first excited state of $\operatorname{Ar}\left(3 s^{2} 3 p^{5} 4 s\right)$ which is shifted to larger energies because of the interaction with neighbouring atoms. The observed shift depends on the strength of the interaction. Ar atoms in the first shell, experiencing a strong interaction with the central Xe atom, show a larger shift than Ar atoms in the second and subsequent shells which interact mainly with each other. This is in accordance with the discussion in the previous paragraph and in agreement with the calculations of Böhmer et al. [19]. The neutral precursor of $\mathrm{Ar}_{7} \mathrm{Xe}^{+}$has only one shell filled, the neutral precursor of $\mathrm{Ar}_{12} \mathrm{Xe}^{+}$consists of at least two shells, which explains the double structure in Fig. 5. The effect of the second shell on the energy shift is less pronounced, i.e. the maximum (or shoulder) should lie closer to the atomic value, which it does.

\section{Conclusions}

From the experiments discussed above the following conclusions can be drawn: Mixed $\mathrm{Ar}$ - Xe-clusters are ionized via three pathways, where two are direct ionization channels and one proceeds via an excited intermediate state of the neutral $\mathrm{Ar}_{n} \mathrm{Xe}$ cluster. The energy released by ionization is partly channelled into fragmentation, which results in the formation of the partcularly stable $\mathrm{Ar}_{12} \mathrm{Xe}^{+}$and $\mathrm{Ar}_{18} \mathrm{Xe}^{+}$ions. At low energies, however, formation of unfragmented metastable cluster-ions can be observed. From the appearance of a pressure dependent maximum around $\mathrm{Ar}_{7} \mathrm{Xe}^{+}$in the size distribution of the ionic clusters it is concluded that the neutral cluster distribution has a particularly strong maximum at $\mathrm{Ar}_{12} \mathrm{Xe}$. This and the shape of the resonance line as a function of cluster size leads us to believe that in both the neutral and the ionic cluster ion, $\mathrm{Xe}$ is the central particle surrounded by shells of Ar atoms. A drastic change in cluster properties is observed after the first shell has been completed. The results are in agreement with recent theoretical calculations.

We thank Dr. U. Böhmer for the help with the experiments in its initial stages and for many valuable discussions. The technical assistance of Mr. J. Lehmann and Mr. M. Fieber, and the assistance with computer programming of Dr. J. Valldorf is greatfully acknowledged.

\section{References}

1. Stern, O.: Z. Phys. 39, 751 (1926); 41, 563 (1927); Naturwiss. 17, 391 (1929); Phys. Rev. 51, 563 (1937);

Knauer, K., Stern, O.: Z. Phys. 39, 764 (1926);

Z. Phys. 39, 780 (1926); Z. Phys. 53, 766 (1929);

Z. Phys. 53, 799 (1929); Z. Phys. 60, 414 (1930)

2. Kantorowitz, A., Grey, J.: Rev. Sci. Instrum. 22, 328 (1951)

3. Anderson, J.B., Andres, R.P., Fenn, J.B.: Supersonic nozzle beams. In: Molecular beams. Ross J. (ed.), p. 275. New York: Interscience 1966

4. Becker, G.W., Bier, K., Henkes, W.: Z. Phys. 146, 333 (1956)

5. Milne, T.A., Greene, F.T., Beachey, J.E.: J. Chem. Phys. 56, 5340 (1972)

6. King, D.L., Dixon, D.A., Herschbach, D.A.: J. Am. Chem. Soc. 96, 3328 (1974)

7. van Lumig, A., Reuss, J.: Int. J. Mass Spectrosc. Ion P. 25, 137 (1977); 27, 197 (1978); 
van Lumig, A., Reuss, J., Ding, A., Weise, J., Rintisch, A.: Mol. Phys. 38, 337 (1979)

8. Lee, G.W., Stein, G.D.: Surf. Sci. 156, 112 (1985)

Farges, J., de Feraudy, M.F., Raoult, B., Torchet, G.: J. Chem. Phys. 78, 5067 (1983)

9. Ceyer, S.T., Tiedemann, P.W., Mahan, B.H., Lee, Y.T.: J. Chem. Phys. 70, 14 (1979)

Ceyer, S.T., Tiedemann, P.W., Ng, C.Y., Mahan, B.H., Lee, Y.T.: J. Chem. Phys. 70, 2138 (1979)

Linn, S.H., Ono, Y., Ng, C.Y.: J. Chem. Phys. 74, 3348 (1981)

10. Takagi, T.: Z. Phys. D-Atoms, Molecules and Clusters 3, 171 (1986)

11. Geraedts, J., Stolte, S., Reuss, J.: Helv. Phys. Acta 56, 529 (1983), Miller, R.E., Watts, R.O., Ding, A.: Chem. Phys. 83, 155 (1984)

12. Haberland, H.: Surf. Sci. 156, 305 (1985)

13. Echt, O., Sattler, K., Recknagel, E.: Phys. Rev. Lett. 56, 1121 (1981)

Echt, O., Reyes-Flotte, A., Knapp, M., Sattler, K., Recknagel, E.: Ber. Bunsenges. Phys. Chem. 86, 860 (1982)

14. Baer, T.: State selection by photoion photoelectron coincidence. In: Gas phase ion chemistry, Vol. 1. New York: Academic Press 1979 ;

Tanaka, K., Kato, T., Guyon, P.M., Koyano, I.: J. Chem. Phys. 77, 4441 (1982);

Guyon, P.M.: Threshold photoion-photoelectron coincidences. In: Electronic and atomic collisions. Oda, N., Takayanagi, K., (eds.). Amsterdam: North Holland 1980

15. Cordis, L., Ganteför, G., HeBlich, J., Ding, A.: Z. Phys. D Atoms, Molecules and Clusters 3, 323 (1986)

16. Radzig, A.A., Smirnow, B.M.: Reference data on atoms, molecules and ions. Berlin, Heidelberg, New York: Springer 1985

17. Heßlich, J., Kuntz, P.J.: Z. Phys. D - Atoms Molecules and Clusters 2, 251 (1986)
Kuntz, P.J., Valldorf, J.: Z. Phys. D - Atoms Molecules and Clusters 8, 195 (1988)

18. Böhmer, H.-U., Peyerimhoff, S.D.: Z. Phys. D - Atoms, Molecules and Clusters 8, 91 (1988)

19. Böhmer, H.-U.: Ph.D. Thesis, Universität Bonn (1988)

20. Ganteför, G., Bröker, G., Holub-Krappe, E., Ding, A.: Proceedings of the SASP'88, p. 331, 1988

21. Ding, A., Cassidy, R.A., Heßlich, J.: Proceedings of the SASP'84, p. 228,1984

22. Ding, A., Futrell, J.H., Cassidy, R.A., Cordis, L., HeBlich, J.: Surf. Sci. 156, 282 (1985)

23. Ding, A., Cassidy, R.A., Futrell, J.H., Cordis, L.: J. Phys. Chem. 91, 2562 (1987)

24. Ng, C.Y.: Adv, Chem. Phys. 52, 263 (1983)

25. Kamke, W., Kamke, B., Kiefl, H.U., Hertel, I.V.: Chem. Phys. Lett. 122, $356(1985)$

26. Ding, A.: Z. Phys. D - Atoms, Molecules and Clusters (accepted)

27. Schwentner, N., Koch, E.-E., Jortner, J.: Electronic excitations in condensed rare gases, p. 33ff. Berlin, Heidelberg, New York: Springer 1985

28. Kolb, D.M., Forstmann, F.: Matrix induced changes in the electronic spectra of isolated atoms and molecules. In: Matrix isolation spectroscopy. Barnes, A.J. et al. (eds.), p. 347-367. Dordrecht: Reidel 1981

29. Echt, O., Sattler, K., Recknagel, E.: Phys. Rev. Lett. 47, 1121 (1981)

30. Märk, T.D., Castleman Jr., A.W.: Adv. At. Mol. Phys. 20, 65 (1985)

31. Bartlett, J.H.: Phys. Rev. 41, 370 (1932);

Elsasser, W.M.: J. Phys. Radium 4, 549 (1933); ibid. 5, 625 (1934) Göppert-Mayer, M.: Phys. Rev. 74, 235 (1948); ibid. 75, 1969 (1949)

32. Ref. 19 , p. $119 \mathrm{ff}$. 